Revue Française de Civilisation Britannique

XXI-2 | 2016

Economic Crisis in the United Kingdom Today: Causes and Consequences

\title{
Réflexions sur la crise
}

Some thoughts on crisis

\section{Stéphane Guy}

\section{(2) OpenEdition \\ Journals}

Electronic version

URL: http://journals.openedition.org/rfcb/1117

DOI: $10.4000 / \mathrm{rfcb} .1117$

ISSN: 2429-4373

Publisher

CRECIB - Centre de recherche et d'études en civilisation britannique

\section{Electronic reference}

Stéphane Guy, «Réflexions sur la crise », Revue Française de Civilisation Britannique [Online], XXI-2 | 2016, Online since 26 September 2016, connection on 19 April 2019. URL : http:// journals.openedition.org/rfcb/1117; DOI : 10.4000/rfcb.1117

This text was automatically generated on 19 April 2019

\section{(c) $(1) \odot$}

Revue française de civilisation britannique est mis à disposition selon les termes de la licence Creative Commons Attribution - Pas d'Utilisation Commerciale - Pas de Modification 4.0 International. 


\title{
Réflexions sur la crise
}

\author{
Some thoughts on crisis
}

\section{Stéphane Guy}

1 Peut-être davantage que d'autres pays, le Royaume-Uni a connu ces dernières années plusieurs crises qui ont eu des conséquences frappantes à l'échelle nationale et internationale. Le référendum sur l'Ecosse et, en juin dernier, celui sur la sortie de l'Europe, ont notamment conduit à la remise en question fondamentale d'institutions, de la politique nationale, de l'identité et des relations internationales dont on ne peut encore mesurer tous les effets à moyen et à long terme. Ce numéro de la Revue Française de Civilisation Britannique propose d'examiner les crises qui ont affecté le Royaume-Uni et leur signification. Celle de 2008 apparaît comme un catalyseur d'autres bouleversements et fait l'objet de plusieurs contributions. Nous avons cependant également invité des articles sur des crises antérieures pour analyser leurs conséquences mais aussi pour tenter de déterminer si le concept même de crise revêt un caractère spécifique dans la sphère britannique.

2 Concept paradoxal, la crise semble constituer un événement à la fois circonscrit dans le temps et récurrent. D'une part, elle marque l'ébranlement d'un système économique de production à un moment donné, et s'accompagne d'une remise en question de certitudes économiques, politiques et sociales qui appellent à leur dépassement. D'autre part, l'histoire même se caractérise par des ruptures, des étapes et des passages qui marquent le caractère répétitif de la crise. Le décès de Margaret Thatcher en 2013 a été l'occasion pour nombre d'observateurs de comparer le tournant libéral qu'on prêta à sa politique et les actions menées par le gouvernement de coalition Cameron-Clegg au pouvoir de 2010 à 2015. Dans les deux cas, il s'agissait de succéder aux Travaillistes à l'issue d'une crise inaugurée par le choc pétrolier de 1973 ou le crash financier en 2007-2008 - et d'affirmer la nécessité de réduire un déficit attribué aux gouvernements sortants. La solution résidait dans la réduction des dépenses publiques. Pour autant, les mesures adoptées ont fait l'objet de nombreuses critiques. Quelques jours avant la journée d'études sur la crise de 2008 organisée par l'Université de Cergy-Pontoise en 2015, le FMI exprimait de fortes réserves sur les politiques d'austérité mises en œuvre au Royaume-Uni, du fait qu'elles risquaient de compromettre la croissance ${ }^{1}$. C'était là un débat ancien qui refaisait surface 
sur les choix à opérer par les pouvoirs publics en matière d'économie et qui traduisait des approches divergentes sur le rôle que pouvaient exercer les pouvoirs publics pour accroître la prospérité du pays ${ }^{2}$. Or précisément cette diversité de réponses proposées en remède à la crise signale son caractère ambivalent. Présentée par les gouvernants comme situation unique résultant d'une politique jugée inefficace ou d'un paradigme devenu caduque, la crise fait en même temps l'objet d'étiologies concurrentes, si l'on reprend le sens médical du terme ${ }^{3}$. Le recul historique tend à agrandir cet écart entre la situation d'urgence utilisée par les gouvernements conservateurs pour justifier un programme et les implications politiques, sociales, idéologiques de leurs actions.

Les études de ce numéro de la Revue Française de Civilisation Britannique montrent comment la crise constitue non seulement un fait économique mais interroge les présupposés de la société où elle survient. Est-il légitime, dans ce cas, de recourir au même terme pour désigner des bouleversements économiques, des changements de gouvernance, des mutations politiques et sociales? Comme le souligne Paul Ricœur dans son étude sur la spécificité moderne de la crise, "une société n'est pas transparente à elle-même; c'est aussi ce que signifie le terme d'idéologie $»^{4}$. En d'autres termes, l'observation de la crise ne saurait être neutre. La crise est une construction conceptuelle qui révèle les modes de pensée et les convictions d'une société à un moment donné. Dès lors, on peut penser que constater une crise, c'est déjà en proposer une résolution. Les articles de la revue permettent d'éclairer les enjeux économiques et politiques de ce concept : explicitant les conséquences des crises des années soixante-dix et de 2008 dans les contextes qui leurs sont propres, ils montrent aussi la manière dont elles cristallisent des contradictions et ambivalences idéologiques et signalent, en définitive, une forme de continuité dans la discontinuité. Il semblerait que les sorties de crises soient aussi diverses et multiples que les interprétations des observateurs.

\section{La crise, un «phénomène social total »}

4 Si elles se comprennent en premier lieu comme problème économique, les crises engendrent des bouleversements qui affectent la société tout entière. La rupture du système de production, le chômage, les incertitudes sur l'avenir conduisent à des remises en question qui débordent la seule sphère économique pour affecter l'équilibre social, l'ordre politique et les convictions mêmes d'une société. À quel point la crise économique pouvait-elle être évitée? Les pouvoirs publics sont-ils responsables des dysfonctionnements? Une politique différente doit-elle être mise en œuvre? Des alternatives réformatrices sont-elles envisageables? Ou bien le remède consiste-t-il en une transformation radicale du système de production, du pouvoir, de l'État? À bien des égards, la crise semble relever de ces " phénomènes sociaux totaux » que désigne Marcel Mauss dans son étude des échanges économiques «Essai sur le don. Forme raison de l'échange dans les sociétés archaïques » (1923-1924) : « ils mettent en branle dans certains cas la totalité de la société et de ses institutions (potlatch, clans affrontés, tribus se visitant, etc.) et dans d'autres cas, seulement un très grand nombre d'institutions, en particulier lorsque ces échanges et ces contrats concernent plutôt des individus. $»^{5}$ 


\section{Un bouleversement du paradigme économique}

5 Les changements qu'entraînent les crises sont perceptibles tout d'abord à l'échelle économique elle-même. Le crash de 2008, par exemple, a conduit à repenser le fonctionnement des marchés financiers : ses causes principales - l'endettement et les opérations à risque des banques - ont été en effet attribuées à l'inefficacité de la supervision et de la réglementation. Ces critiques ont débouché sur des réformes importantes à l'échelle internationale et plus spécifiquement britannique pour tenter de contenir les excès des spéculateurs, limiter la création de produits toxiques ou encore surveiller les pratiques des établissements bancaires.

6 Le Royaume-Uni figure parmi les pays qui ont mis en œuvre des changements considérables. Marie-Claude Esposito examine ainsi la refonte du système bancaire par le renforcement de la supervision, la mise en place d'une réglementation plus contraignante que celle existant avant 2008, et, allant plus loin que les recommandations de Bâle III, la séparation dans un même établissement des opérations de banque de détail et de banque d'investissement. Le système tripartite qui confiait la réglementation financière entre le Financial Services Authority, la Treasury et la Bank of England (BoE) fut, par exemple, accusé d'inefficacité par les Conservateurs qui, une fois élus, firent le choix de renforcer les pouvoirs de la banque centrale britannique.

7 L'orthodoxie économique visant à contenir l'inflation a également été battue en brèche par les pouvoirs publics avec la politique d'expansion monétaire mise en œuvre dès 2009 avec le Quantitative Easing ou l'abaissement des taux d'intérêt, qui atteignirent 0,5\% en mars $2009^{6}$. Alors que, depuis la crise des années 1970, les gouvernements britanniques tendaient à vouloir séparer la sphère économique de la sphère politique, notamment avec la dérèglementation de 1986 et l'indépendance de la BoE de 1997, la Grande Dépression de 2008 conduit les pouvoirs publics à rattacher la lutte contre l'inflation à des considérations conjoncturelles telles que l'emploi ou la croissance. L'article de Nathalie Champroux fait état, en particulier, d'une réforme de la politique monétaire qui, sans le remettre en cause fondamentalement, a adapté le cadre fondé sur le ciblage de l'inflation pour assurer une « meilleure coordination entre les politiques monétaire et financière ".

8 Les excès de l'industrie financière ayant été unanimement condamnés, les questions éthiques se sont posées de manière plus aiguë. En 2012, le gouvernement de coalition conservateur et libéral-démocrate met ainsi en place le «Big Society Capital» qui a pour vocation d'aider les sociétés de financement à but social à lever des fonds, marquant la volonté de réconcilier la finance, la société civile et l'État : emblématique du programme électoral des Conservateurs menés par David Cameron alors qu'ils étaient dans l'opposition, la «Big Society» consistait à promouvoir les initiatives de la société civile comme alternative à l'État-providence. Les manquements du marché ont donné lieu à des initiatives comparables dont l'article d'Aymen Boughanmi s'efforce de montrer les enjeux : prenant appui sur des sociétés qui ont vu le jour avant ou après le tournant de 2008, l'auteur interroge la notion de finance éthique pour en souligner les atouts (préservation de l'environnement, réduction des inégalités sociales, pragmatisme) autant que les ambiguïtés et les limites. En tout état de cause, ces initiatives signifient, pour l'auteur, que les chocs économiques peuvent donner lieu non pas à l'effondrement du marché mais à son renouvellement. 
9 De manière plus générale, les crises peuvent entraîner des coupes budgétaires se traduisant par une réduction des dépenses publiques à destination nationale ou internationale. Ce fut le cas lorsque le Department for International Development annonça en novembre 2012 la décision controversée de supprimer l'aide au développement pour l'Inde ${ }^{7}$, et pour l'Afrique du Sud en avril $2013^{8}$. L'aide au développement, qui a fait du Royaume-Uni en mars 2013 le premier pays du G8 à tenir ses engagements sur l'allocation de $0,7 \%$ du revenu intérieur brut à ce poste, semble pourtant faire figure d'exception ${ }^{9}$. On sait que le gouvernement néo-travailliste de Tony Blair avait mis en place le DFiD en 1997 en vue de réduire la pauvreté des pays en voie de développement par la promotion de la croissance et de l'emploi. Il s'agissait par là-même aussi de contribuer à la stabilité et à la sécurité sur le plan international. La coalition ne semble pas avoir remis en cause de manière radicale les principes ni les politiques de ce Ministère. Ondine Aza souligne l'augmentation régulière des contributions britanniques depuis les gouvernements Blair jusqu'au gouvernement de coalition - alors même, on peut le préciser, que la politique d'austérité rendait certains Conservateurs critiques à l'égard du maintien des dépenses consacrées à l'Official Development Aid (ODA), ou "Aide Publique au Développement ». L'auteur de l'article met cependant en valeur la complexité des facteurs en jeu. D'une part, l'augmentation du budget alloué au développement a été revue à la baisse, de $38 \%$ prévue en 2010-2011 à 27 \% en 2014-2015, soit une réduction de 1246 millions de livres sterling. D'autre part, il semble que les prêts soient de plus en plus destinés à remplacer les dons. Enfin, la Coalition a voulu corréler, davantage que ne l'avaient fait les Travaillistes sous Blair et Brown, les aides aux enjeux de stabilité et de sécurité.

\section{Ruptures conservatrices et chocs sociaux : des remèdes libéraux ?}

10 Si elles conduisent à des réformes du marché et de la politique économique, les crises économiques peuvent aussi donner lieu à des bouleversements politiques et sociaux. La récession, l'inflation excessive ou la déflation, le chômage et le déficit budgétaire peuvent en effet être attribués à une mauvaise gestion de l'économie par les pouvoirs publics d'un pays plutôt qu'à des facteurs exogènes ou à la conjoncture internationale. La crise peut alors entraîner une rupture politique et une crise sociale. Dans l'opposition comme au pouvoir, les Conservateurs ont reproché aux Travaillistes ce qu'ils jugeaient être leur étatisme et leur imprudence budgétaire («tax-and-spend»), et les ont à ce titre désignés comme responsables des crises qui ont notamment précédé les gouvernements Thatcher ou Cameron. Ainsi ont-ils mis en œuvre des politiques qui se voulaient fondamentalement différentes des précédentes.

\section{Le tournant des années soixante-dix}

11 La révolution néo-libérale de Thatcher trouve ses origines dans les effets sociaux de la stagflation qui conduisent à la remise en question du consensus social-démocrate d'aprèsguerre. Alimentée par le choc pétrolier de 1973 mais résultant également des pressions salariales qui se font plus conflictuelles depuis les années soixante, l'inflation galopante s'accompagne de grèves de plus en plus nombreuses et amples pendant les années 1970 qui paralysent le pays : la Dame de Fer peut alors mettre en œuvre une rupture radicale avec l'État corporatiste et la culture de l'assistanat attribués au travaillisme. La réponse néo-libérale de Thatcher à la stagflation revêt ainsi une dimension culturelle et idéologique autant qu'économique : la combinaison inédite de l'inflation et du chômage 
défiait en effet les lois économiques qui semblaient avoir régi les sociétés occidentales depuis la Seconde Guerre mondiale. Représentée par la courbe de Philips (1958), la loi selon laquelle le taux d'inflation aurait dû être inversement proportionnel à celui du chômage, était invalidée par l'expérience. Sans qu'on parvînt à identifier clairement les causes de ce phénomène, c'était le modèle fordiste qui était mis à l'épreuve : l'idéal d'une croissance illimitée se heurtait à la stagnation de la productivité et rendait les revendications salariales de plus en plus difficiles à satisfaire. Un signe précurseur de cette impasse fut l'échec de la réforme qu'Harold Wilson tenta de mener en 1969 avec le livre blanc In Place of Strife pour contenir les hausses de salaires et qui, face à l'opposition de l'aile gauche du parti travailliste et des syndicats, dut être suspendue. Au-delà des enjeux salariaux et du manque de structure des syndicats, lequel explique en partie pourquoi un compromis ne put être trouvé, ce divorce entre la classe ouvrière et le travaillisme peut se lire comme la conséquence d'évolutions économiques et sociales dont les grèves des années 1970 furent le symptôme mais qui échappaient aux analyses traditionnelles.

12 La politique monétariste que mena le premier gouvernement Thatcher avec le « Medium Term Financial Strategy" se voulait une rupture avec une conception keynesienne de l'économie défendue par les Travaillistes autant que par les Conservateurs majoritaires au sein du parti lorsqu'elle parvint à sa direction en 1975. Par opposition avec les politiques de gestion de la demande et de contrôle des prix et des salaires ${ }^{10}$, la volonté inspirée par Milton Friedman de lutter contre l'inflation en réduisant la quantité de monnaie en circulation restait encore au début des années 1980 en porte-à-faux avec la pensée universitaire au Royaume-Uni : ainsi l'augmentation des impôts prévue par le budget de 1981 dans un contexte de forte récession donna lieu à la publication d'une lettre qui en contestait l'efficacité et qui fut signée par trois-cent-soixante-quatre économistes ${ }^{11}$. Quels qu'aient été ses résultats, cette rupture avec un keynesianisme encore majoritaire dans l' establishment s'apparente à l'émergence d'un nouveau paradigme libéral qui devait se traduire par des effets sociaux et culturels et marquer le passage du Royaume-Uni à une dérèglementation assumée de l'économie.

\section{Cameron face à la crise : I'héritage thatchérien}

La réaction de David Cameron à la crise se présente de manière comparable. L'imprévoyance d'un État travailliste jugé dépensier est invoquée pour justifier les coupes budgétaires et la cure d'austérité mis en œuvre à partir de 2010 : « they didn't fix the roof when the sun was shining $»^{12}$. Au lieu de tenter de réduire le déficit pendant la période de prospérité où ils étaient au pouvoir, les Travaillistes auraient accru de manière irresponsable les dépenses en matière de santé et d'éducation ainsi que la dépendance des individus à l'égard de l'état, au détriment de l'esprit d'entreprise. Les Conservateurs attribuent au déficit les effets catastrophiques de la crise au Royaume-Uni et affirment à ce titre la nécessité de réduire drastiquement les dépenses publiques pour rétablir l'équilibre budgétaire et préserver la crédibilité de l'économie britannique sur les marchés ${ }^{13}$. La crise fournit un arrière-plan pour justifier l'application d'un programme politique susceptible d'être impopulaire mais présenté comme inévitable remède.

Tout le propos de Simon Lee, dans son article « Developmental State or Developmental Market? The Cameron-Clegg government's Response to The Financial Crisis », vise à éclairer et à nuancer cette rupture politique que prétendirent promouvoir à la fois la 
Coalition, en réponse à la crise de 2008, et les gouvernements Thatcher, suite aux troubles des années soixante-dix. Contrairement à ce que laisse penser la critique de l'étatisme qui émaille leur discours, leur politique s'inscrit en réalité dans une conception « développementiste » de l'État, selon la terminologie de Chalmers Johnson qui fait de celui-ci, plutôt que le marché, l'instrument de la croissance ${ }^{14}$. Simon Lee met en lumière cette tradition britannique qui remonte au dix-septième siècle pour battre en brèche l'affirmation du gouvernement de coalition selon laquelle l'austérité constituerait le seul moyen, «necessary and inevitable », de réduire le déficit.

Ne serait-ce que par leur étendue et leur impopularité, les mesures d'austérité participent aussi d'une crise sociale. Pour rétablir l'équilibre budgétaire, le gouvernement a multiplié les réductions d'allocations qui se traduisent par une diminution des revenus des ménages, un nouveau train de mesures plus contraignantes ayant été adopté après les élections de 2015. De fait, la contestation de cette politique a pris la forme de manifestations de grande ampleur qui, le 23 juin 2014 et le 16 avril 2016, comptaient plus de cinquante mille participants. Parmi les nombreuses personnalités hostiles à cette orientation du gouvernement, l'archevêque catholique de Westminster Vincent Nichols reprochait par exemple à la Coalition, dans un entretien paru dans le Telegraph le 14 février 2014, de plonger dans « la faim et la misère » un nombre croissant de personnes, qualifiant de «honteux» («a disgrace ») le programme d'austérité mis en œuvre et de «punitif» le système de prestations sociales nouvellement en vigueur ${ }^{15}$. De ce point de vue, la crise a conduit les gouvernements Cameron à une rupture critique avec les principes travaillistes du distributivisme qui, selon certains observateurs, se traduisent par une croissance des inégalités. Raphaëlle Espiet-Kilty tente ainsi de prouver comment l'augmentation de la pauvreté est due non pas à la crise économique en tant que telle mais au remède d'austérité administré par les gouvernements Cameron: la montée des prix de l'immobilier, la précarisation de l'emploi et l'endettement des ménages, se conjuguent avec la réduction des prestations sociales, qui «semble avoir joué rôle prépondérant ». Pour l'auteure, «ces restrictions budgétaires ne résultent pas de la crise mais relèvent d'un choix idéologique ", la Big Society servant à mettre en œuvre un programme qui, au nom de la société civile, ambitionne de réduire les dépenses publiques ainsi que l'État-Providence.

\section{Le paradigme libéral à l'épreuve de la crise}

16 La crise de 2008 a enfin entraîné une remise en question du paradigme libéral. L'une des causes de la Grande Dépression, on le sait, fut la prolifération de produits financiers toxiques et le surendettement dont les États-Unis furent l'exemple le plus manifeste, la contagion s'expliquant en partie par la financiarisation du capitalisme occidental. Jusqu'à l'éclatement de la crise, la demande avait pu être soutenue artificiellement par l'excès de crédit sans que les taux de profit ne semblent baisser. Cette incohérence a pu donner lieu à une remise en cause par l'opinion de la libéralisation des services financiers et de la prépondérance de la place occupée par les banques. Or, les gouvernements occidentaux ne paraissent pas disposés à tenir compte de ces interrogations au point d'envisager une réorientation radicale de la politique économique. Malgré un pouvoir de contrôle accru sur l'administration des établissements en question, l'intervention des pouvoirs publics pour éviter la faillite des banques semblait cautionner leurs abus. En annonçant le 17 février 2008 la nationalisation de Northern Rock ${ }^{16}$, celle de Bradford and Bingley le 29 septembre ${ }^{17}$ et, le 13 octobre, un plan de sauvetage consistant à injecter trente-sept 
milliards de livres dans les établissements bancaires Royal Bank of Scotland, Lloyds TSB et $\mathrm{HBOS}^{18}$, le gouvernement Brown entendait stabiliser le système financier et éviter une dépression économique mais en appelait aux contribuables britanniques pour réparer les excès des banques ${ }^{19}$. Les regrets exprimés par le Premier ministre travailliste en avril 2010 à propos d'un défaut de réglementation pouvaient apparaître bien peu convaincants aux yeux des observateurs pour qui les pratiques contestables du secteur financier demandaient une rupture avec le néo-libéralisme ${ }^{20}$.

La lenteur de la reprise depuis 2008 tend à faire penser que les efforts en direction des banques au détriment des ménages conduisent à une sous-consommation et à une crise de la croissance, le crédit ne permettant plus de la doper comme auparavant. Assiste-t-on à une redéfinition du libéralisme? Les ouvrages de Thomas Piketty, Le capital au vingt-etunième siècle (2013), ou de Joseph Stiglitz, par exemple Freefall. America, Free Markets and the Sinking of the World Economy (2010), développent des analyses qui le laissent entendre. A cet égard, la crise de 2008 a ravivé une pensée hostile à l'économie de marché. Nicholas Sowels recourt ainsi à la pensée de Marx pour tenter de démontrer que les politiques menées depuis 2008 n'ont pas fourni de réponse satisfaisante ni équitable aux dysfonctionnements du marché faute d'avoir mis en œuvre sa modification fondamentale : pour l'auteur, le secteur bancaire est plus concentré qu'auparavant, la politique du quantative easing a été inefficace et inégalitaire, et les taux bas n'ont pas permis la croissance escomptée. De manière comparable aux années 1970, avec les solutions libérales imposées en réaction à la crise, le capitalisme deviendrait de plus en plus "conflictuel ", facteur de divisions sociales importantes, sans être en mesure de créer des richesses réelles. La crise économique occasionne donc un choc non seulement politique et social mais idéologique.

\section{De la crise à la critique : la politisation d'un concept}

De manière plus générale, les défaillances du marché peuvent alimenter les oppositions partisanes et conduire à une forme de fracture dans le débat politique. Les relations de l'individu avec l'État sont en effet conditionnées en partie par la production et la distribution des richesses. L'effondrement de l'industrie ou de la finance entraîne alors une critique de gouvernants jugés inaptes à défendre l'intérêt général.

Découlant de la crise de 2008, la politique d'austérité a alimenté, par exemple, le nationalisme écossais et exacerbé les tensions institutionnelles. Les avancées du Scottish National Party s'expliquent en partie, dès le début des années soixante-dix, par la découverte des ressources pétrolières, qui, selon les membres du SNP, assureront la prospérité. Elles tiennent aussi à la désapprobation, chez une majorité d'Écossais, de la gestion de la crise par le gouvernement conservateur : l'austérité sert alors les arguments en faveur de la rupture institutionnelle prônée par le SNP. Edwige Camp-Pietrain s'efforce de démontrer comment celui-ci, ainsi que les autres partis écossais, « utilisent, et même instrumentalisent, les questions économiques et sociales, en particulier la crise économique et financière. » Mettant en lumière les paradoxes et les ambiguïtés des formations politiques, elle souligne notamment comment le SNP entend se présenter comme un rempart contre l'austérité tout en étant hostile à une redistribution par la fiscalité : il affiche des politiques "sociales-démocrates côté dépenses et néo-libérales côté recettes». Aux yeux de certains observateurs, malgré les réformes sociales que permettraient une rupture avec le Royaume-Uni, la crise peut apparaître comme 
catalyseur d'un projet indépendantiste dont la viabilité économique est sujette à controverse, alors même que semblent sous-utilisées les compétences offertes par la dévolution.

Marc Lenormand souligne comment, pendant les années 1970, le discours de crise s'inscrit dans un "arsenal rhétorique » que déploient, paradoxalement, tout autant les néolibéraux, encore minoritaires au sein du parti conservateur que l'aile gauche du parti travailliste. Qu'ils invoquent l'accroissement ou la réduction du rôle de l'État dans l'économie, ces courants remettent en cause le consensus d'après-guerre qui aurait atteint un point de saturation et rendrait "nécessaire » la mise en œuvre de politiques révolutionnaires à partir d'un nouveau paradigme, socialiste ou libéral. Pour confirmer la thèse de l'auteur, on pourrait rappeler comment la crise semble constitutive de la stratégie électorale de celle qu'on en vint à désigner par l'acronyme TINA (« There Is No Alternative $»)^{21}$. C'est d'ailleurs cette justification de politiques radicales par la crise que l'on retrouve quelque quarante ans plus tard chez David Cameron lors de son discours sur l'économie du 7 mars 2014 : «We are making tough choices about our future. But we are making the right choices. If there was another way I would take it. But there is no alternative $»^{22}$.

Les défaillances du marché marquent donc le débat politique en amplifiant les oppositions existantes. Dans cette perspective, Gino Raymond examine dans quelle mesure la révolution thatchérienne a véritablement constitué une rupture idéologique. Dans « The 1970s and the Thatcherite Revolution: Crisis of Ideology or Control? », il tente d'éclairer le thatchérisme à la lumière de la postmodernité : les failles et les incohérences que l'on trouve dans les programmes et les politiques des Conservateurs menés par Thatcher comme des Travaillistes manifestent un émiettement des certitudes et des récits totalisants, la révolution thatchérienne se traduisant par une substitution de la communication politique à l'idéologie.

La manière dont la crise affecte le débat politique pose donc la question du concept luimême. Une rupture absolue est-elle réalisable, étant donné que, bien qu'exprimant des opinions contraires, les individus font partie d'un même groupe social, partagent des valeurs et des représentations communes et évoluent dans un même contexte historique ? Peut-on constater l'effondrement d'un paradigme et en proposer un autre en étant soi-même affecté par la crise ? James Connelly s'efforce de dénouer ces paradoxes inhérents au concept de crise. Empruntant à Thomas Kuhn sa terminologie, l'auteur souligne que la " politique normale » se traduit par une administration rationnelle, une expertise et des institutions qui ne permettent pas la résolution des problèmes fondamentaux tels que le rapport entre écologie et économie. Pour autant, la «politique révolutionnaire » ne peut pas non plus prétendre s'affranchir entièrement d'un système existant : «Just as anomalies only make sense against the background of a paradigm, so a paradigm only makes sense against a background of science, that is, a particular tradition, per se ». Arrière-plan commun, la tradition ne peut être évitée ni par les Conservateurs, ni par les révolutionnaires, la crise masquée - un effondrement logique d'un système qui peut ne pas être identifié sur-le-champ par les observateurs - devant être distinguée de la crise perçue -l'expérience psychologique ou intellectuelle d'une incompréhension et d'une perte de repères. Pour James Connelly, ce sont, en définitive, la dialectique et la reconceptualisation qui permettent de penser la crise.

On le voit, la notion de crise pose problème. D'une part, la crise désigne un événement ou une rupture. D'autre part, le fait même d'employer ce concept suppose une capacité à 
résoudre les contradictions du moment. Ainsi la crise apparaît-elle moins comme un phénomène paroxystique que comme l'étape d'un processus de développement.

\section{Les limites de la crise : de la rupture à la continuité}

\section{La permanence du choix politique} une ligne de conduite. Même s'ils invoquent une situation d'urgence pour présenter une action comme unique possibilité, on peut constater que des multitudes de choix s'offrent aux responsables politiques. Davantage qu'un effondrement ou une destruction, la crise constitue alors, au sens grec de krisis, un moment décisif qui permet aux gouvernants d'exercer leur jugement et leur discernement politique. Comme le souligne Reinhart Koselleck dans son étude de 1982 sur la crise en lien avec les concepts fondamentaux de l'histoire: " "crisis" was a central concept by which justice and the political order ( Herrschaftsordnung) could be harmonized through appropriate legal decisions. $»^{23}$

La crise bancaire, par exemple, a conduit à la réaffirmation du pouvoir politique alors que la sphère économique semblait être devenue autonome. Depuis les années quatre-vingts, la réglementation avait tendu à être dissociée des gouvernements. Fléau des années cinquante et soixante et caractéristique des "stop-go policies ", la manipulation des taux d'intérêts à des fins électoralistes avait en effet fait l'objet de nombreuses critiques inaugurées par Thatcher. Le monétarisme semblait alors constituer une solution à la crise de gouvernance en confiant la politique économique aux banques centrales sans mandat électoral ${ }^{24}$. Cette "dépolitisation" s'exprima en particulier sous Tony Blair avec l'indépendance accordée à la Bank of England en 1998 qui donnait au Monetary Policy Committee la responsabilité exclusive de fixer les taux d'intérêt. La refonte du système bancaire qu'analyse Marie-Claude Esposito marque une approche distincte de cette logique : le renforcement des pouvoirs de contrôle accordés à la Bank of England et la suppression de la Financial Services Authority qui en était autrefois indépendante, répondaient à la volonté affichée de «redonner à la Banque d'Angleterre à la fois le contrôle macro-prudentiel et le contrôle micro-prudentiel ». Les réformes engagées à la suite de la crise visent donc à clarifier les responsabilités que le système tripartite avait été accusé d'effacer ${ }^{25}$.

Le choix et le discernement n'ont pas été non plus entamés par la crise de 2008 en matière de politique monétaire. La volonté de contenir l'inflation depuis les années 1980 s'est en effet traduite par une souplesse quant à l'application des objectifs. D'une part, souligne Nathalie Champroux, il s'agissait pour Thatcher comme pour Major d'un moyen et non d'un but: "they said monetary stability was a pre-condition, but was not sufficient to ensure economic growth and employment.» Avec la crise, cette conception a été reformulée plus explicitement. Les orientations britanniques en matière monétaire se conforment de fait à une tendance perceptible à travers le monde qui tend à légitimer les choix politiques en fonction de la conjoncture :

A worldwide recurring recommendation was that of spreading the use of FWG, with a clear statement about future policy intentions linked to specific economic conditions, whether these conditions were still anomalous or back to normal. Another repeated instruction was that of keeping monetary policy as a last line of defence, but securing coordination between monetary and macro-prudential policies.

Revue Française de Civilisation Britannique, XXI-2 | 2016 
Il semble donc que la crise n'ait pas limité les choix politiques mais qu'elle ait au contraire confirmé l'existence des possibilités qui s'offrent aux pouvoirs publics et aux autorités monétaires de prendre en compte le contexte industriel et social pour mettre en œuvre la réduction de l'inflation. C'est bien d'ailleurs cette souplesse qui, rappelle Nathalie Champroux, était revendiquée dès 1997 par le Gouverneur de la Bank of England Mervyn King à propos des banques centrales: «He explained that... their role was to properly choose which time horizon was the best to return inflation to target without too much damage on output.» Loin de s'opposer à la délibération des gouvernants, le principe de lutte contre l'inflation peut se traduire par des appréciations différentes du rythme auquel elle sera mise en œuvre. Selon l'importance accordée à la production, les délais d'application seront plus ou moins proches. Si l'on suit l'auteure, la réforme de la politique monétaire n'a fait qu'officialiser une situation antérieure à 2008 : l'essentiel du changement tient ainsi plutôt à la réaffirmation d'un pouvoir décisionnaire, ou " discrétion ", déjà détenu par les autorités. Indépendamment de la crise, on constate une résilience du choix politique.

De fait, malgré ses défaillances, le marché n'a pas empêché les acteurs économiques d'inventer des alternatives aux abus et aux excès spéculatifs, en vue d'une répartition plus juste des richesses, ce qui a conduit Marx à les juger comme obstacles au socialisme. Ainsi le mutualisme, les friendly societies et les coopératives se sont-ils développés au dixneuvième siècle alors même que le laissez-faire semblait faire autorité. La finance éthique elle-même ne date pas de la crise. Intégrée à la législation dès 2000 par le parlement britannique, avant la formulation par l'ONU des principes du Socially Responsible Investment en $2006^{26}$, elle s'inscrit dans une tradition contractualiste qui remonte à plusieurs siècles et se manifeste par de multiples initiatives émanant de la société civile, dont, par exemple, les sociétés de crédit immobilier qu'étudie Aymen Boughanmi. L'affirmation de l'auteur selon laquelle « les succès de la finance dite éthique constituent une nouvelle démonstration de l'extraordinaire capacité de l'idéologie libérale à s'adapter aux différentes circonstances " pourrait, de fait, s'appliquer à toutes les entreprises qui se font jour en contrepoint du capitalisme financier et que désignent aujourd'hui les concepts d'économie solidaire, d'économie participative ou de micro-crédit. Des tentatives semblables ont existé depuis les débuts du capitalisme dès lors que, par exemple, la classe ouvrière a pu se forger une identité et une culture spécifiques, ainsi que l'a souligné E. P. Thompson dans The Making of the English Working Class (1963), créant des structures et des organisations qui lui permettaient de contourner l'oppression économique exercée par le capitaliste. Il semblerait donc que l'existence de pratiques abusives dans le secteur bancaire ne rende nécessaire ou inévitable ni une remise en question fondamentale de l'économie de marché, ni la protection complaisante des intérêts financiers : les individus hostiles aux excès du libéralisme peuvent choisir de se joindre à des initiatives privées portées vers l'intérêt général au sein d'un cadre favorable aux principes de concurrence et de profit.

\section{La résilience du libéralisme économique}

Ces initiatives montrent donc que les crises économiques n'entraînent pas la disparition de l'économie de marché. Une première constatation peut être d'ordre électoral : malgré l'impopularité des mesures prises par les gouvernements Thatcher et Cameron-Clegg dès leur premier mandat, les Conservateurs ont été reconduits lors des législatives de 1983 et 
de 2015. On peut attribuer ces victoires aux imperfections du scrutin majoritaire uninominal, aux circonstances du moment - la guerre des Malouines scella une forme d'unité patriotique autour de la Dame de Fer - ou aux discours catastrophistes de George Osborne qui parviennent à convaincre une partie de l'opinion de la nécessité de maintenir le cap de l'équilibre budgétaire : il demeure que l'option libérale n'a pas été véritablement rejetée par une majorité d'électeurs dans le cadre considéré comme démocratique. ${ }^{27}{ }^{2}$ 'est précisément cette pérennité de la tradition libérale que met en valeur l'article de Simon Lee lorsqu'il affirme que la participation "développementiste» de l'État à l'expansion économique se retrouve au dix-septième siècle et dans les politiques thatchériennes aussi bien que dans les orientations de la coalition Cameron-Clegg:

The state has simply defended the interests and promoted the same sectors of the economy-financial services and military industries-which it has always done ever since England's financial, political, scientific, administrative revolutions during the seventeenth century English Enlightenment.

Distincte du laissez-faire, cette tradition manifeste une préférence récurrente, et peutêtre constante, des pouvoirs publics pour le marché plutôt que la redistribution ou la justice sociale.

31 De manière comparable, Raphaelle Espiet-Kilty et Nicholas Sowels établissent une correspondance étroite entre les politiques néolibérales de Thatcher et de Cameron, le second jugeant qu'il n'y a pas eu de changement notable d'orientation politique depuis les années 1980, y compris sous les gouvernements travaillistes Blair et Brown. Pour Raphaelle Espiet-Kilty, les coupes budgétaires sont en cohérence avec le programme conservateur antérieurement aux politiques de David Cameron : il s'agit d'un choix qui « restait fondamentalement idéologique. La cible principale de ces réductions avait été identifiée bien avant 2008. » La crise, selon l'auteure, « peut difficilement être vue comme autre chose qu'un prétexte ». Si l'on suit ce raisonnement, les politiques néolibérales sont idéologiques parce qu'elles ne procèdent pas seulement d'un programme électoral mais qu'elles s'ancrent dans une tradition qui n'est pas consciemment articulée. En dépit des inégalités amplifiées par l'austérité, les mesures annoncées comme moyens de réduire le déficit public et de rassurer les marchés continuent à être adoptées par des gouvernements issus d'élections démocratiques. Peut-être inhérente à l'évolution des économies occidentales vers un capitalisme financier, la volonté de préserver le secteur bancaire de la faillite procède sans doute d'une hégémonie, justifiée ou non, de l'économie de marché: on peut penser que la crise de 2008 n'a pas entrâné de changement majeur dans la finance parce que les gouvernements Brown et Cameron ont agi en conformité avec les principes libéraux antérieurs. Ainsi, pour Nicholas Sowels: "The response of the UK authorities - in line with authorities throughout the industrialised world was, in short, to pursue a policy of getting back to business as usual...as quickly as possible.» Avant de proposer une explication de la crise, l'auteur conclut que, sans être parvenues à surmonter les difficultés rencontrées, les décisions des pouvoirs publics s'inscrivent dans une continuité remarquable :

In short, there is a good case to be made that if the (re-)regulation of banking has been significantly enhanced, the way banks and markets operate has not been changed fundamentally. Significantly too, the financial crisis has left the sector even more concentrated than before, both globally and in the UK.

Les effets des crises économiques sont enfin limités parce qu'elles ne font que manifester de multiples ruptures d'autres ordres. Si tous les observateurs s'accordent à voir dans les grèves et la paralysie du pays ou dans l'effondrement des cours boursiers et les faillites 
d'établissements bancaires le signe d'un échec, les causes des dysfonctionnements sont, elles, sujettes à des interprétations aussi nombreuses que contradictoires. En définitive, de même que la crise marque l'écroulement d'un système ou son point de rupture, le constat de défaillances économiques signale une crise du sens. Parce qu'elle cristallise des opinions contradictoires, la crise est inhérente à l'usage même de la raison et devient permanente. Ainsi que le souligne Myriam Revault d'Allonnes, « la modernité elle-même est intrinsèquement habitée par la crise. Et pour l'homme contemporain qui vit le déchirement des valeurs, la crise est ce vécu lui-même $»^{28}$.

\section{La permanence du conflit}

33 Le nationalisme et l'indépendantisme écossais sont bien antérieurs à la crise de 2008. Si celle-ci a pu précipiter les tensions au point de rendre possible l'organisation du référendum en 2014, le principe d'une Écosse séparée du Royaume-Uni s'ancre dans des lectures opposées de leur passé. Au nationalisme qui affirme que l'Écosse vit sous le joug de l'Angleterre depuis la fusion des deux parlements en 1701, symbolisé par le poème de Robert Burns, "Such a parcel of rogues in a nation" (1791), s'oppose l'interprétation, explicitée plus récemment par Linda Colley, dans Britons. Forging the Nation (1992), par exemple, selon laquelle les deux nations auraient retiré un bénéfice partagé de l'union sur les plans économique, social et politique. La cure d'austérité décidée par le gouvernement Cameron-Clegg en réaction à la crise ne fait donc que cristalliser un débat séculaire entre les deux nations sur la souveraineté de l'Écosse. D'où la multitude d'interprétations, par exemple, sur la viabilité économique de l'indépendance que souligne Edwige Camp-Pietrain dans son article. La crise pourrait-elle être surmontée par les ressources pétrolières? Sans conteste, jugeaient les indépendantistes lors des débats de la campagne référendaire. A l'inverse, « les unionistes, réunis au sein de Better Together , faisaient une tout autre lecture des mêmes données.» On peut en effet prolonger le propos de l'auteure en soulignant que ces perceptions et démonstrations différentes procèdent d'un clivage culturel et politique qui, avant la crise de 2008, séparait déjà l'électorat écossais, traditionnellement ancré à gauche, d'une Angleterre à majorité conservatrice pendant les années 1980 et 1990.

Cette conflictualité des opinions que reflète la crise se manifeste particulièrement à propos de l'histoire. Pour les observateurs qui recourent au terme même de "crise ", il s'agit en effet non pas seulement de constater des souffrances ou des malheurs contemporains mais aussi de développer une chaîne de causalité qui en rende compte. Cette étiologie peut donner lieu, par conséquent, à des interprétations contradictoires, à des périodisations divergentes, à des raisonnements antagonistes. Ainsi en est-il du consensus d'après-guerre que la révolution néo-libérale de Thatcher serait venue mettre à mal. Selon cette interprétation, elle aurait appliqué des politiques radicales en réaction aux pressions salariales exercées par les syndicats et au déficit budgétaire. Bien qu'en partie historiquement fondé - les politiques de la Dame de Fer ont consisté en une libéralisation du marché, une privatisation de nombre d'entreprises publiques, une lutte persistante contre les syndicats -, le caractère révolutionnaire du thatchérisme semble devoir être nuancé à plusieurs titres.

Tout d'abord, l'idée même de consensus ne fait pas l'unanimité chez les historiens, comme le montrent les études de Harriet Jones et de Michael Kandiah parues dans The Myth of Consensus (1996). Ainsi que l'affirme par exemple Kandiah : 
the conservative leaders did not share the assumptions which underpinned Labour' $\mathrm{s}$ vision of postwar Britain; they were determined to construct and then maintain Conservatism as a distinct and viable alternative to Labour's socialism, and they wished to preserve and promote capitalism and maintain limited social welfare programmes within the context of increasing economic growth ${ }^{29}$.

Par ailleurs, les principes énoncés par Thatcher n'ont pas été appliqués de manière systématique et cohérente. Alors qu'elle avait toujours affirmé promouvoir la souveraineté nationale, elle ratifia ainsi sans hésiter l'Acte Unique de 1986 qui donnait davantage de pouvoirs supranationaux aux institutions européennes. Quand bien même les conséquences de l'accroissement du vote à la majorité qualifiée lui auraient échappé ce qu'elle affirme dans ses mémoires de 1993 -, on peut penser que ce soutien procédait aussi d'une volonté de promouvoir le capitalisme et de lutter contre l'État corporatiste précisément au moyen des institutions. C'est cette ambivalence que met en évidence, sur le plan idéologique, Simon Lee lorsqu'il souligne comment Hayek lui-même en vint à préconiser dans Law, Legislation and Liberty (1973) un État fort pour faire triompher les principes libéraux sur les intérêts particuliers: «the basic principles of a free society might have to be "temporarily suspended when the long-run preservation of that order is itself threatened" ". En outre, en matière de politique économique, les principes monétaristes inspirés de Milton Friedman et de Friedrich Hayek ont été contredits par la politique d'expansion monétaire que poursuivit le Chancelier de l'Échiquier Nigel Lawson et que toléra Thatcher, ${ }^{30}$ ou encore par le budget généreux de 1983. À ces incohérences s'ajoutent celles évoquées par Gino Raymond dans son article, la lutte contre le déficit s'étant accompagnée d'un chômage qui fit croître le nombre d'allocataires et donc les dépenses publiques.

Affirmer que «l'hiver du mécontentement » rend inévitables les politiques libérales est aussi sujet à discussion, comme le montre Marc Lenormand lorsqu'il indique que la crise a été interprétée simultanément comme celle du marché ou de l'État. Sa réflexion et celle de Gino Raymond révèlent comment les interprétations contradictoires de la période de l'après-guerre interrogent l'existence même d'un compromis d'après-guerre.

Enfin, bien que minoritaires quand Thatcher prit la direction du parti en 1975, les idées libérales avaient déjà été expérimentées par Edward Heath pendant la première partie de son mandat. En affirmant, dans sa formule célèbre, « you turn if you want to - the lady's not for turning", elle lui reprochait moins les principes guidant sa politique que son renoncement face aux syndicats. D'autres études ont pu montrer que l'échec de Heath tenait au manque de cohérence idéologique de son libéralisme mais aussi sans doute aux circonstances internationales, dont le choc pétrolier de 1973, et à son incapacité à séduire l'électorat des classes moyennes en contrepartie de coupes opérées dans les subventions et les prestations sociales.

Ces lectures divergentes montrent donc que les crises signalent des dysfonctionnements passagers et conjoncturels mais aussi des divergences de vue propres à l'esprit critique. Soumis à l'examen rationnel, le réel est l'objet d'interprétations conflictuelles et d'étiologies concurrentes. Le constat collectif de dysfonctionnements, d'injustices ou de souffrances manifeste des systèmes de pensée et des croyances aussi divers que les observateurs eux-mêmes. Au lieu d'être passagère, la crise devient donc le symptôme permanent de la modernité. Dans ce sens, il s'agit moins d'un bouleversement que d'un moment décisif où les individus sont appelés à porter un jugement sur le monde. 


\section{Quelles sorties de crise ? La critique comme discernement}

\section{La mise en perspective historique contre le prétexte de la nécessité}

L'un des remèdes à la rhétorique de l'urgence et au discours de la nécessité immédiate qui se dessine dans ce recueil d'articles est l'Histoire. Convoquée à des fins idéologiques, la crise est en effet présentée comme une situation extraordinaire qui ne laisse aucun choix aux gouvernants dans la mise en œuvre d'une politique. La réflexion sur le passé permet au contraire de relativiser les solutions invoquées: loin d'être un problème isolé qui appelle un remède unique, les bouleversements constatés à un moment donné sont comparables à des situations antérieures. Face à l'idéologie qui se caractérise par un système unique d'interprétation du réel et évite le débat démocratique, l'Histoire permet une critique du discours de crise qui ouvre le champ des possibles.

41 Selon Simon Lee, par exemple, la politique du gouvernement Cameron-Clegg n'a rien de nécessaire et s'inscrit dans la tradition d'un État «développementiste » qui met les pouvoirs publics au service du marché. Le sauvetage des banques par les gouvernements néo-travailliste et conservateur-libéral s'explique donc par un conformisme intellectuel et théorique que l'étude de l'histoire permet de mettre au jour :

modern British politics has become stranded on the ideological common ground of the political economy of neo-liberalism, which has itself been founded upon an alternative political narrative and very particular understanding of the history of England and the United Kingdom.

Pour Lee, l'identification de l'idéologie néo-libérale passe par la déconstruction du « récit politique » qui fait du laissez-faire la cause et le modèle des succès économiques. La sortie de crise exige donc un retour sur le passé grâce auquel l'observateur pourra distinguer les choix idéologiques de ceux dictés par l'urgence, comme l'indique la conclusion de l'auteur à propos de l'affectation des sommes déboursées par la Treasury: "If the cost of that intervention and set of priorities is prolonged austerity for millions of ordinary citizens and those on low incomes or disabled, then so be it. »

Pour Nicholas Sowels, l'histoire constitue également le moyen de démontrer l'inefficacité et l'incohérence du capitalisme. Loin de la surmonter, les politiques des gouvernements Thatcher et Cameron-Clegg auraient non seulement engendré la crise mais lui auraient permis de persister. Les inégalités sont en effet la cause d'une chute de la demande qui entretient les difficultés économiques rencontrées par les entreprises. Si, selon la thèse de T. H. Marshall à laquelle souscrit l'auteur, les droits et les acquis sociaux se sont développés à partir des années 1950, l'augmentation des inégalités depuis la fin des années 1970 et l'effondrement du consensus d'après-guerre illustre le fait que la politique économique des gouvernements occidentaux, et notamment britanniques, répondait moins aux crises qu'elle ne les alimentait. C'est l'étude du passé qui permet à Nicholas Sowels d'affirmer que le néolibéralisme constitue une «rupture de l'histoire» et que le capitalisme n'a pas engendré la prospérité annoncée: « historically wealth tends to be much more concentrated than income, and this too is a situation which has been worsened by liberalism ». Une solution à la crise consiste donc à déconstruire l'idéologie capitaliste par un décloisonnement de l'économie: il s'agit de rattacher la science économique à des données sociales et politiques. Le recours à l'histoire permet ainsi, 
selon la perspective marxiste adoptée par l'auteur, de proposer des alternatives à l'économie dominante ou « mainstream ».

Une manière complémentaire de démontrer le caractère idéologique des mesures gouvernementales consiste à souligner la continuité avec des programmes passés. Les Travaillistes et les Conservateurs ont, indépendamment de la crise, cherché à réduire les compétences de l'État au profit de la société civile. La décision de réduire les prestations sociales s'explique moins par les circonstances extraordinaires de la crise que par une logique qui se perpétue d'un gouvernement à l'autre. Au caractère exceptionnel et urgent des mesures invoqué par la Coalition pour justifier sa politique d'austérité, Raphaëlle Espiet-Kilty oppose ce qu'elle juge être un courant remontant à Edmund Burke : « La crise ne fait que confirmer un projet déjà inscrit et qui repose sur une vision conservatrice traditionnelle de la relation entre l'État et la société civile ». Le recours à l'histoire permet donc d'interroger le discours de crise pour souligner la dimension non pas seulement économique mais idéologique des mesures adoptées : la sortie de crise passe alors par un éclatement de la perspective restreinte et immédiate de gouvernants focalisés sur le seul choc économique et par une complexification du problème grâce à la prise en compte des dimensions sociale et politique des remèdes proposés. Pour dénouer la crise sociale, l'auteure de l'article entend ainsi s'extraire du paradigme économique en adoptant une vision plus globale: «La question est de savoir si des facteurs, autres que simplement conjoncturels, peuvent expliquer cette augmentation ». Reste à savoir si la continuité des politiques et des idées mises en avant par les auteurs suffit à les qualifier de traditions métahistoriques. Dans quelle mesure la critique salutaire que constitue la désignation d'incohérences et de contradictions peut-elle se conjuguer à la proposition de remèdes plus équitables ou efficaces? Enfin, la déconstruction du récit idéologique ne risque-t-elle pas aussi d'être elle-même sous-tendue par un autre récit selon lequel la crise constituerait le révélateur ultime de déséquilibres ou d'injustices sociaux. Pour Karl Löwith, une telle philosophie de l'histoire participerait d'une théologie sécularisée qui apparait notamment dans le marxisme :

Le processus entier de l'histoire, tel qu'il est exposé par Le Manifeste, reflète le schéma général de l'interprétation judéo-chrétienne de l'histoire comme advenir providentiel du Salut orienté vers un ultime accomplissement du sens. Le matérialisme historique est une histoire sacrée formulée dans la langue de l'économie politique ${ }^{31}$.

\section{L'analyse critique du discours politique}

La crise peut aussi être surmontée grâce à l'identification non pas d'un métarécit mais des incohérences du discours politique. Le constat de la crise repose en effet sur un système d'interprétation du monde qui peut lui-même s'apparenter à une forme d'idéologie : le réel est intégré à une grille de lecture qui se veut à la fois diagnostic et pronostic, et qui risque par conséquent d'être présentée comme panacée par des gouvernants soucieux de gagner l'adhésion des électeurs. C'est précisément la fonction de la science politique que d'analyser les déclarations et les programmes en prenant en compte les rapports de force et d'influence qui existent entre les individus, la situation des partis et l'évolution des mentalités à une période donnée, les stratégies de persuasion et le fonctionnement des institutions. Une critique montrant les failles d'un programme permet alors de dépasser l'idéologie de crise et la séduction dangereuse qu'elle peut exercer. 

les partis écossais révèle l'écart entre la théorie et la pratique. Chacun d'entre eux déploie une justification argumentée de l'indépendance ou de l'union qui intègre notamment les mêmes données économiques relatives à la crise en vue de rallier l'opinion à l'une ou l'autre position. La mise en regard critique des actions et des discours permet donc de dépasser les circonstances du moment pour envisager les intérêts et les luttes de pouvoir qui sous-tendent les débats. Ainsi l'auteure souligne-t-elle comment, sous la direction de Nicola Sturgeon, le SNP a pu faire évoluer son projet de réduction systématique de l'impôt sur les sociétés en vue de s'assurer un soutien travailliste, "lui préférant des baisses ciblées ». Elle rappelle encore comment les "politiques emblématiques» mises en avant par le gouvernement SNP, telles que la suppression d'inscription dans l'enseignement supérieur, s'accompagnent, selon l'opposition, de coupes opérées ailleurs et moins visibles, comme celle du montant des bourses universitaires. Les programmes politiques, selon cette approche, ne constituent nullement un bloc défini par des principes économiques anhistoriques ni une idéologie immuable mais sont en évolution constante et soumis à des facteurs partisans, institutionnels, sociologiques, voire psychologiques. Distincts du traité théorique, le discours politique est modelé par des stratégies complexes. Tout l'objet de la science politique sera alors de mettre en perspective l'événement unique que constitue la crise pour montrer, selon les termes de l'auteur, «le décalage entre la rhétorique du SNP, efficace pour gagner les élections, et son bilan au gouvernement écossais depuis 2007, moins flamboyant», ou, en d'autres termes, de distinguer le réel de son interprétation.

Dans son étude sur la révolution thatchérienne, Gino Raymond développe également une critique du discours politique qui souligne l'écart entre les idées énoncées par Margaret Thatcher et ses actions. La stratégie de communication et le travail sur l'image se substituent, pour Raymond, au débat d'idées et à la confrontation d'idéologies : «Her impact did not come from the eloquence of her discourse, but from the soundbites and well-turned phrases written for her, the perfectly timed put-down, and above all the work of the image-makers to whom she willingly submitted. » Que ce phénomène date, ou non, de la période où Thatcher dirigea le parti (l'expansion des médias et de la télévision pendant les années soixante n'a peut-être qu'amplifié ces éléments essentiels de la politique que sont la persuasion et la séduction de l'électorat), la mise en regard du discours et des politiques menées permet le dépassement de l'idéologie de crise.

De la même façon, le discours de crise procède de stratégies politiques et de luttes d'influence qui conduisent à revêtir le réel de significations politiques. Mettre celles-ci en évidence permet une distance critique avec le caractère prétendument urgent et inévitable des remèdes invoqués. L'article de Marc Lenormand montre ainsi la construction du discours politique dans son contexte : la divergence des interprétations qui prétendent identifier le système dont la crise marquerait l'effondrement signale la nécessité de prendre en compte la situation d'énonciation, les paramètres qui permettent au discours de prendre forme et d'être reçu favorablement par l'électorat. S'appuyant sur l'analyse du récit que développe Colin Hay dans son article «Narrating crisis: The Discursive Construction of the Winter of Discontent $»^{32}$, l'auteur signale par exemple le rôle joué par les médias pendant les mois précédant l'arrivée de Margaret Thatcher au pouvoir, qui « construisent un contraste entre d'un côté un gouvernement insouciant et de l'autre le désir d'action de Margaret Thatcher ». Marc Lenormand cite aussi, comme Gino Raymond, l'absence d'opposition intellectuelle dans le Parti travailliste, ou encore 
les facteurs qui ont conduit Thatcher à la tête du parti, comme plus déterminants que le discours de crise lui-même. construction idéologique qu'un état de fait objectif. Selon les termes de Harriet Jones :

the consensus paradigm was emerging in the late 1970s and 1980s, at a time when Thatcherism was the defining force of political debate. The chasm between the values and approaches of the two major parties was never greater than it was after 1979 , inevitably influencing analysis of the early postwar period. It seemed that the basis for any common ground between the parties had irrevocably broken down, and that contrast with what had gone before provided both an obvious and fascinating focus for contemporary historians ${ }^{33}$.

e paradigme $d u$ consensus naît d'une situation spécifique où s'opposent deux convictions politiques et qui marque la lecture du passé de son empreinte. De la même façon, la crise peut se comprendre comme projection d'un affrontement idéologique. En contextualisant les principes et les arguments énoncés par les gouvernants, la science politique permet de relativiser le caractère exceptionnel des problèmes à résoudre et de montrer que la perception même de la crise est conditionnée par des enjeux de pouvoir.

\section{Le choix du consensus}

51 Une autre manière de surmonter la crise consiste enfin à s'accorder sur un certain nombre de principes qui détermineront une ligne de conduite acceptée par une majorité. C'est ce consensus qui a conduit les puissances occidentales à adopter des mesures similaires pour tenter d'éviter une dépression économique occasionnée par le crash financier. Les réponses des gouvernements Brown et Cameron au déficit, par exemple, signalent une certaine convergence de vues. Comme l'affirme Andrew Gamble en reprenant les observations de divers commentateurs, qu'il s'agisse des Conservateurs ou des Travaillistes, «no party was prepared to advocate a thorough-going Keynesian approach to the deficit, which would have been to make no plans to cut the deficit at all until the recovery was firmly established $\aleph^{34}$. La ligne de fracture politique concernait davantage une question de délais que de principe: "In Britain in 2009-2010, the argument centered on timing. Over what period should the budget deficit be eliminated?», ${ }^{35}$ les Travaillistes jugeant nécessaire mais moins urgent que les Conservateurs un équilibre budgétaire. Les gouvernements qui ont dû résoudre l'épreuve du crash financier semblent donc avoir partagé une même acceptation du marché qui les a conduits à verser d'importantes sommes pour les banques britanniques et soutenir l'économie. Cette confiance commune dans un système libéral apparaît avec les réformes du secteur bancaire exposées par Marie-Claude Esposito.

Les reproches adressés par l'opposition conservatrice aux gouvernements travaillistes concernaient en effet notamment l'incapacité du système de surveillance bancaire à identifier et à éviter les dérives. Pour autant, la Coalition comme les gouvernements qui l'avaient précédée partageaient une même conviction selon laquelle une forme de réglementation du marché financier était nécessaire. De fait, les faillites telles que celle de la Barings en 1995 avaient conduit à la mise en place de garde-fous. En d'autres termes, c'est la forme de la réglementation qui fait l'objet de divergences plutôt que sa justification en tant que telle: le principe du marché est accepté en même temps que celui d'établir des règles évitant les excès de spéculation, le gouvernement Brown ayant lui-même, au lendemain de la crise, émis des propositions pour améliorer la stabilité 
financière. Selon cette perspective, la sortie de crise passe par des réformes institutionnelles qui reposent à la fois sur un rejet de l'auto-régulation du marché et sur la croyance dans la capacité des institutions à protéger l'intérêt général.

Pour d'autres observateurs, c'est l'adoption par les autorités monétaires d'une orientation générale qui permettra une sortie de crise. L'article de Nathalie Champroux montre comment, plutôt qu'un dogme immuable, le ciblage de l'inflation s'est accompagné de « déviations » répétées qui traduisaient la volonté des pouvoirs publics d'associer la politique monétaire à des enjeux économiques plus larges. Au-delà de ces divergences, la mise en place d'un cadre fixant les limites à ne pas dépasser signale une volonté partagée de contenir l'inflation afin d'assurer la croissance. Par contraste avec un objectif purement arithmétique déterminé par un pouvoir technocratique, le cadre permet assez de souplesse pour une adaptation en fonction des circonstances et reconnait donc aux gouvernements démocratiquement élus une forme de responsabilité et d'initiative. La crise ne ferait alors que créer les conditions où le pouvoir politique est amené à décider.

Selon une autre perspective, la crise peut être surmontée si les acteurs économiques s'accordent sur le bien commun et l'intérêt général. La création de richesses par le marché se conjuguera alors à des questions d'ordre moral ou éthique que formuleront les acteurs économiques. Un engagement contractuel pourra par exemple être scellé en conformité avec les principes de justice, d'écologie ou de développement durable qui auront été identifiés au préalable et approuvés par les parties, indépendamment ou en complément de leurs seuls intérêts économiques. Les pratiques abusives qui ont engendré la crise peuvent alors être dépassées par une prise de conscience de l'intérêt général et la volonté partagée de promouvoir une société plus juste dans le cadre d'une économie libérale. L'article d'Aymen Boughanmi offre des réflexions qui vont dans ce sens. Partagée entre la nécessité de faire des profits inhérente à toute entreprise commerciale et la poursuite par des individus d'idéaux ou de principes, la finance éthique constitue une réponse viable à la crise si les acteurs économiques parviennent à trouver « le sens de la cohésion sociale ». Pour les établissements de prêt, il s'agira par exemple d'éviter d'imposer des taux élevés aux personnes déjà fortement endettées.

Dans tous ces cas, la sortie de crise suppose la détermination collective de principes vers lesquels les activités économiques doivent tendre. Selon cette perspective, il convient de remettre en cause l'autonomie de la sphère économique pour envisager la crise également dans ses dimensions sociale, politique et éthique ou morale.

Un autre exemple de la sortie de crise par le consensus se dessine enfin avec la question de l'aide au développement. On l'a noté plus haut, l'article d'Ondine Aza montre comment le maintien du soutien apporté par le Royaume-Uni aux pays pauvres ne semble pas avoir souffert de la crise parce qu'une multitude de facteurs ont été pris en compte tels que la sécurité et la stabilité internationale, ainsi que les causes de la pauvreté plutôt que la seule pauvreté elle-même. C'est donc une vision globale ou holiste du développement qui permet la pérennisation de la contribution financière, vision qui nécessite la coopération de plusieurs ministères au-delà des coûts immédiats que représente l'aide au développement. L'auteur souligne ainsi que le Department for International Development fondé par les Travaillistes en 1997 doit en partie sa réussite au fait que ses politiques ont été menées en collaboration avec d'autres départements: "They managed to find a common ground for their different interests and this contributed to strengthening the role and position of the DfID ». Au concept humanitaire qui risquait de devenir lettre 
morte par excès d'idéalisme s'est ajoutée une réflexion pratique avec les ministères concernés, ce qui a assuré la pérennité de l'ODA. C'est d'ailleurs l'exploitation de cette collaboration évoquée par Ondine Aza que propose l'étude de l'Overseas Development Institute de mai 2012, The UK's Approach to Linking Development and Security: Assessing Policy and Practice. En plus d'une identification plus spécifique des enjeux de politique intérieure propres à chaque bénéficiaire de l'aide, les auteurs recommandent une concertation accrue entre les ministères :

Thirdly, this is now a key moment to substantively realise cross government coordination. The creation of specific mechanisms - such as the Conflict Pools or Stabilisation Unit - has not overcome differences in organisational culture and approach across UK government departments. But collaboration now needs to be within the DNA of departments who need to work effectively in conflict countries. Wherever possible, context analysis should be shared by DFID, FCO, MOD and others ${ }^{36}$.

La sortie de crise exige donc, selon ces analyses, une collaboration entre les différentes parties qui suppose une croyance dans les possibilités offertes par la politique de résoudre les problèmes que rencontre une société. Il s'agit alors en premier lieu de partager une conviction sur l'aptitude de la communauté humaine à établir un ensemble de valeurs ou de principes qui détermineront les actions à mener en faveur du bien commun, ce qui rejoindrait les conclusions de Paul Ricœur :

On ne peut ici que parier et espérer. Mon propre pari et mon propre espoir sont que, en dépit de l'absence de consensus et de conviction forte dans notre société pluraliste, une chance inédite est ouverte au renouvellement des héritages du passé : une double chance de ressourcement et de réinterprétation ${ }^{37}$.

Stéphane Guy est Maître de Conférences à l'université de Cergy-Pontoise et membre du laboratoire AGORA. Ses recherches portent sur l'histoire des idées et la pensée politique du dix-neuvième siècle à nos jours. Il a notamment publié des articles sur les intellectuels et le socialisme anglais ainsi que sur la Société Fabienne, et codirigé avec Catherine Marshall The Victorian Legacy in Political Thought (Peter Lang, 2014).

\section{BIBLIOGRAPHY}

Bank of England, «Statistical Interactive Database », <http://www.bankofengland.co.uk/ boeapps/iadb/Repo.asp> [consulté le 20 juin 2016].

BBC News, « UK Banks Receive £37bn Bail-Out », BBC News, 13 octobre 2008, <http:// news.bbc.co.uk/2/hi/business/7666570.stm> [consulté le 20 juin 2016].

Bingham John, « New Cardinal Vincent Nichols: welfare cuts 'frankly a disgrace' », The Daily Telegraph, 14 février 2014, <http://www.telegraph.co.uk/news/religion/10639015/New-CardinalVincent-Nichols-welfare-cuts-frankly-a-disgrace.html> [consulté le 20 juin 2016].

Cameron David, « Budget Response Speech », 22 avril 2009, <http://www.telegraph.co.uk/ finance/budget/5203479/David-Camerons-Budget-response-speech-in-full.html>, [consulté le 20 juin 2016]. 
Cameron David, « Economy Speech », 7 mars 2013, <https://www.gov.uk/government/speeches/ economy-speech-delivered-by-david-cameron> [consulté le 20 juin 2016]

Cook Chris et Sked Alan, Post-war Britain : A Political History (Londres, Penguin, 1993).

Darling Alistair, déclaration du 17 février 2008, reproduite in Financial Times, 17 février 2008, <http://www.ft.com/cms/s/0/bda727f6-dd7f-11dc-ad7e-0000779fd2ac.html\#axzz4E5UftzMo> [consulté le 20 juin 2016].

Floud Roderick, Humphries Jane, Johnson Paul (ed.), The Cambridge Economic History of Modern Britain, vol. 2, (Cambridge, Cambridge University Press, 2014).

Gamble Andrew, « The United Kingdom: The Triumph of Fiscal Realism ? », in Grant Wyn etWilson Graham K. (ed.), The Consequences of the Global Financial Crisis. The Rhetoric of Reform and Regulation, (Oxford, OUP, 2012), pp. 34-50.

GOV. UK, «India: Greening announces new development relationship », 9 novembre 2012, < https://www.gov.uk/government/news/india-greening-announces-new-developmentrelationship>, [consulté le 20 juin 2016].

GOV.UK, «UK to end direct financial support to South Africa », <https://www.gov.uk/ government/news/uk-to-end-direct-financial-support-to-south-africa>, [consulté le 20 juin 2016].

Green Jeremy, Hay Colin, Taylor-Gooby Peter (ed.), The British Growth Crisis. The Search for a New Model (Londres, Palgrave, 2015).

Hay Colin, « Narrating Crisis: The Discursive Construction of the Winter of Discontent ", Sociology, vol. 30, $\mathrm{n}^{\circ}$ 2, 1996, pp. 253-277.

H. M. Treasury,déclaration reproduite in The Guardian, 29 septembre 2008, <https:// www.theguardian.com/business/2008/sep/29/bradfordbingley.banking4> [consulté le 20 juin 2016].

Jay Antony (ed.), Oxford Dictionary of Political Quotations (Oxford, Oxford University Press, 2012), <http://www.oxfordreference.com/view/10.1093/acref/9780199572687.001.0001/qauthor-00002-00001503> [consulté le 20 juin 2016].

Johnson Chalmers, MITI and the Japanese Miracle. The Growth of Industrial Policy, 1925-1975 (Stanford, Stanford University Press, 1982).

Jones Harriet et Kandiah Michael (eds.), The Myth of Consensus (Londres: Palgrave, 1996).

Koselleck Reinhart et Richter Michaela W., « Crisis », Journal of the History of Ideas, Vol. 67, No. 2 (avril 2006),

Löwith Karl, Histoire et salut : les présupposés théologiques de la philosophie de l'histoire (Paris, Gallimard, 2002), p. 70.

Mauss Marcel, « Essai sur le don. Forme et raison de l'échange dans les sociétés archaïques ", in Sociologie et anthropologie (Paris, PUF, 1991).

Mulholland Hélène, et Wintour Patrick, « Gordon Brown admits banks needed more regulation », The Guardian, 14 avril 2010, <http://www.theguardian.com/politics/2010/apr/13/gordon-brownbanking-sector-regulation> [consulté le 20 juin 2016].

Ping Chan Zhu, « UK Can Afford to Live with High Debt, IMF Study Insists », The Daily Telegraph, mercredi 3 juin 2015. 
Osborne George, « Speech by the Chancellor of the Exchequer, Rt Hon George Osborne MP, to the Davos CBI British Business Leaders Lunch », 27 janvier 2012, <https://www.gov.uk/government/ speeches/speech-by-the-chancellor-of-the-exchequer-rt-hon-george-osborne-mp-to-the-davoscbi-british-business-leaders-lunch> [consulté le 20 juin 2016].

Provost Claire et Tran Mark, « Aid: how much does the UK spend, why it's important and how it works ", The Guardian, 20 mars 2013, <https://www.theguardian.com/global-development/2013/ mar/20/uk-aid-spend-important-works> [consulté le 20 juin 2016].

Revault d'Allonnes Myriam, «Comment la crise vient à la philosophie », Esprit 2012/3 (Mars/ avril), pp. 108-117.

Ricœur Paul, « La crise : un phénomène spécifiquement moderne ? », Revue de théologie et de philosophie $\mathrm{n}^{\circ} 120$ (1988).

Robinson Nick, « Economy: There is No Alternative (TINA) is Back », BBC News, 7 mars 2013, <http://www.bbc.com/news/uk-politics-21703018> [consulté le 20 juin 2016]

Wild Leni et Elhawary Samir, The UK's Approach to Linking Development and Security: Assessing Policy and Practice, (Overseas Development Insitute, mai 2012), <https://www.odi.org/sites/odi.org.uk/ files/odi-assets/publications-opinion-files/7658.pdf> [consulté le 20 juin 2016].

Winnett Robert et Wallop Harry, « Financial crisis: Bradford \& Bingley Nationalisation Will Cost Taxpayers £150bn », The Daily Telegraph, 28 septembre 2008, <http://www.telegraph.co.uk/ finance/financialcrisis/3098279/Financial-crisis-Bradford-and-Bingley-nationalisation-will-costtaxpayers-150bn.html> [consulté le 20 juin 2016].

\section{NOTES}

1. PING CHAN Zhu, «UK Can Afford to Live with High Debt, IMF Study Insists », The Daily Telegraph, mercredi 3 juin 2015.

2. La polémique entre le FMI et le gouvernement de coalition n'était pas nouvelle. Ainsi que le souligne Ben CLIFT: "The key dissonance between the IMF mission and the UK government during 2013 Article IV consultations, over a number of billion pounds of infrastructure spending, reveals deeper differences about the public power to positively affect economic outcomes ». Ben CLIFT, «The UK Macroeconomic Policy Debate and the British Growth Crisis: Debt and Deficit Discourse in the Great Recession ", in GREEN Jeremy, HAY Colin, TAYLOR-GOOBY Peter (ed.), The British Growth Crisis. The Search for a New Model (Londres, Palgrave, 2015), p. 169.

3. Sur les sens du concept de "crise ", KOSELLECK Reinhart et RICHTER Michaela W., "Crisis", Journal of the History of Ideas, Vol. 67, No. 2 (avril 2006), pp. 357-400.

4. RICÆUR Paul, «La crise : un phénomène spécifiquement moderne?", Revue de théologie et de philosophie $\mathrm{n}^{\circ} 120$ (1988), p. 15.

5. MAuss Marcel, « Essai sur le don. Forme et raison de l'échange dans les sociétés archaïques », in Sociologie et anthropologie (Paris, PUF, 1991), p. 274.

6. BANK OF ENGLAND, «Statistical Interactive Database", <http://www.bankofengland.co.uk/ boeapps/iadb/Repo.asp> [consulté le 20 juin 2016].

7. GOV. UK, «India: Greening announces new development relationship », 9 novembre 2012, < https://www.gov.uk/government/news/india-greening-announces-new-developmentrelationship>, [consulté le 20 juin 2016].

8. GOV.UK, « UK to end direct financial support to South Africa », <https:// www.gov.uk/government/news/uk-to-end-direct-financial-support-to-south-africa $>$, [consulté le 20 juin 2016]. 
9. PROvOST Claire et TRAN Mark, « Aid: how much does the UK spend, why it's important and how it works ", The Guardian, 20 mars 2013, <https:// www.theguardian.com/global-development/2013/mar/20/uk-aid-spend-importantworks> [consulté le 20 juin 2016].

10. Floud Roderick, HUMPHRIES Jane, JOHNSON Paul (ed.), The Cambridge Economic History of Modern Britain, vol. 2, (Cambridge, Cambridge University Press, 2014), p. 463.

11. Ibid., p. 524.

12. CAMERON David, «Budget Response Speech », 22 avril 2009, <http://www.telegraph.co.uk/ finance/budget/5203479/David-Camerons-Budget-response-speech-in-full.html>, [consulté le 20 juin 2016].

13. GAMBLE Andrew, "The United Kingdom: The Triumph of Fiscal Realism?", in GRANT Wyn et WILson Graham K. (ed.), The Consequences of the Global Financial Crisis. The Rhetoric of Reform and Regulation, (Oxford, OUP, 2012), pp. 34-50.

14. JoHnson Chalmers, MITI and the Japanese Miracle. The Growth of Industrial Policy, 1925-1975 (Stanford, Stanford University Press, 1982).

15. BINGHAM John, « New Cardinal Vincent Nichols: welfare cuts 'frankly a disgrace' ", The Daily Telegraph, 14 février 2014, <http://www.telegraph.co.uk/news/ religion/10639015/New-Cardinal-Vincent-Nichols-welfare-cuts-frankly-adisgrace.html> [consulté le 20 juin 2016]. 16. DARLING Alistair, déclaration du 17 février 2008, reproduite in Financial Times, 17 février 2008, <http://www.ft.com/cms/s/0/bda727f6-dd7f-11dcad7e-0000779fd2ac.html\#axzz4E5UftzMo> [consulté le 20 juin 2016]. 17. Déclaration de la TREASURY, reproduite in The Guardian, 29 septembre 2008, <https://www.theguardian.com/business/2008/sep/29/bradfordbingley.banking4> [consulté le 20 juin 2016].

18. BBC, « UK Banks Receive £37bn Bail-Out », BBC News, 13 octobre 2008, <http://news.bbc.co.uk/2/hi/business/7666570.stm> [consulté le 20 juin 2016].

19. WINNETT Robert et WALLOP Harry, « Financial crisis: Bradford \& Bingley Nationalisation Will Cost Taxpayers £150bn », The Daily Telegraph, 28 septembre 2008,

$<$ http://www.telegraph.co.uk/finance/financialcrisis/3098279/Financial-crisis-Bradford-andBingley-nationalisation-will-cost-taxpayers-150bn.html> [consulté le 20 juin 2016].

20. MULholland Hélène, et wintour Patrick, « Gordon Brown admits banks needed more regulation ", The Guardian, 14 avril 2010, <http://www.theguardian.com/politics/2010/apr/13/gordon-brown-bankingsector-regulation> [consulté le 20 juin 2016].

21. Cet acronyme lui aurait été attribué à la suite d'une conférence prononcée devant le Congrès des Femmes Conservatrices le 21 mai 1980: «We get our production and our earnings in balance. There's no easy popularity in what we are proposing, but it is fundamentally sound. Yet I believe people accept there is no real alternative. » in JAY Antony (ed.), Oxford Dictionary of Political Quotations (Oxford, Oxford University Press, 2012),

<http://www.oxfordreference.com/view/10.1093/acref/9780199572687.001.0001/qauthor-00002-00001503> [consulté le 20 juin 2016].

22. CAMERON David, "Déclaration sur l'économie», 7 mars 2013, < https://www.gov.uk/ government/speeches/economy-speech-delivered-by-david-cameron>. Les échos thatchériens de cette formulation n'échappent naturellement à aucun observateur. Voir par exemple l'analyse de 
l'éditorialiste politique Nick ROBINSON : « Economy: There is No Alternative (TINA) is Back », BBC News, 7 mars 2013, <http://www.bbc.com/news/uk-politics-21703018> [consulté le 20 juin 2016]. 23. KOSELLECK Reinhart et RICHTER Michaela W., «Crisis », op. cit., p. 359.

24. GRANT et WILSON (ed.), The Consequences of the Global Financial Crisis, op. cit., p. 4.

25. Voir le discours de George OSBORNE devant le Confederation of British Industry à Davos, le 27 janvier 2012 : «The tripartite structure was incoherent, without clear lines of accountability. The tripartite committee didn't meet for almost a decade. Everyone was so focused on ticking off a regulatory check-list that nobody felt it was their responsibility to use their judgement", <https://www.gov.uk/government/speeches/speech-by-the-chancellor-of-the-exchequer-rthon-george-osborne-mp-to-the-davos-cbi-british-business-leaders-lunch> [consulté le 20 juin 2016].

26. Selon le Pension Disclosure Regulation (2000), les fonds de pensions devaient divulguer la manière dont leur stratégie d'investissement prenait en compte des critères sociaux, environnementaux et de gouvernance.

27. GRANT et WILSON (ed.), The Consequences of the Global Financial Crisis, op. cit., p.13.

28. ReVAult D’Allonnes Myriam, «Comment la crise vient à la philosophie », Esprit 2012/3 (Mars/avril), p. 115.

29. JONES Harriet et KANDIAH Michael (eds.), The Myth of Consensus (Londres: Palgrave, 1996), p.74.

30. соок Chris et SKED Alan, Post-war Britain : A Political History (Londres, Penguin, 1993).

31. LÖWITH Karl, Histoire et salut: les présupposés théologiques de la philosophie de l'histoire (Paris, Gallimard, 2002), p. 70.

32. HAY Colin, "Narrating Crisis: The Discursive Construction of the Winter of Discontent", Sociology, vol. 30, n² 2, 1996, pp. 253-277.

33. JONES Harriet et KANDIAH Michael, The Myth of Consensus, p. xiv.

34. GAMBLE, p. 41.

35. GAMBLE, p. 41.

36. WILD Leni et ELHAWARY Samir, The UK's Approach to Linking Development and Security: Assessing Policy and Practice, (Overseas Development Insitute, mai 2012), p. 11, <https://www.odi.org/sites/ odi.org.uk/files/odi-assets/publications-opinion-files/7658.pdf> [consulté le 20 juin 2016].

37. RICœUR Paul, op. cit., p. 16.

\section{AUTHOR}

\section{STÉPHANE GUY}

Université de Cergy-Pontoise, AGORA

fr 